International Journal of Pure and Applied Mathematics

Volume 107 No. 2 2016, 317-330

ISSN: 1311-8080 (printed version); ISSN: 1314-3395 (on-line version)

url: http://www.ijpam.eu

doi: 10.12732 /ijpam.v107i2.3

\title{
SYNCHRONIZATION OF A PLANAR MAGNETIC BINARIES PROBLEM WHEN THE PRIMARIES ARE OBLATE SPHEROIDS
}

\author{
Mohd. Arif ${ }^{1}$, Ravi Kumar Sagar ${ }^{2} \S$ \\ ${ }^{1}$ Department of Mathematics \\ Zakir Husain Delhi College \\ University of Delhi \\ Delhi, INDIA \\ hmohdarif@gmail.com ${ }^{2}$ Department of Mathematics \\ University of Delhi \\ Delhi, INDIA
}

\begin{abstract}
In this article we have investigated the synchronization behavior of the planar magnetic-binaries problem by taking into consideration the oblateness of the primaries evolving from different initial conditions using active control technique based on the Lyapunovstability theory and Routh-Hurwitz criteria. Numerical simulations are performed to plot phase portraits, time series analysis graphs of the master system and the slave system which further illustrate the effectiveness of the proposed control techniques.
\end{abstract}

AMS Subject Classification: 70F07, 70F15, 34D06

Key Words: magnetic binary problem, synchronization, Lyapunov stability theory, RouthHurwitz criteria

\section{Introduction}

In 1990, two different discoveries changed the point of view of the field of nonlinear dynamics, in particular the point of view of the chaotic behaviour, leading to a new perspective. Firstly Ott, Grebogi and Yorke introduced the possibility of

Received: November 26, 2015

Published: April 4, 2016

${ }^{\S}$ Correspondence author
(C) 2016 Academic Publications, Ltd.

url: www.acadpubl.eu 
controlling chaos [1], then secondly Pecora and Carroll explored the possibility of synchronization of chaotic system [2]. Chaos synchronization has been extensively studied due to its potential applications in real-world complex problems. Many methods and techniques for chaos control and synchronization of various chaotic systems have been developed, such as non linear feedback control [3] , OGY approach [1], sliding mode control [4], adaptive synchronization [5], anti synchronization method [6], active control [7] and so on.

Active control method, proposed by Bai and Lonngren [7] is simple and easy to implement in practical application of synchronization. The method is attractive amongst researchers as it does not require Lyapunov exponents or derivative of the controller while implementing in synchronization problems. It has been successfully used to synchronize two identical as well as non identical chaotic system $[6,8]$.

Stormer(1907) [9] has studied the motion of a charged particle moving in the field of a magnetic dipole as a two body problem. This problem in general is quite complicated and is non integrable. A. Mavraganis has studied the motion of a charged particle moving in the field of two rotating magnetic dipoles instead of one dipole.

In 1977, Mavraganis [10] laid the foundation of the magnetic binary problem, that is, the study of charged particle behavior in a magnetic binary system and the stationary state investigation but it did not take into consideration the oblateness of each one of the primaries. After this study, Mavraganis [11] investigated the influence of the primaries with oblateness on the stationary state of the planar magnetic binary problem. Recently Arif [12] studied the motion of charged particle in magnetic binary system when the primaries are oblate spheroids with the shifting of one primary to the origin. Khan and Shahzad [13] have studied the problem of synchronization of circular restricted three body problem which is similar to magnetic binaries problem ( restricted three body problem with magnetic moment of primaries ).

In this paper, we have applied the active control technique based on the Lyapunov stability theory and Routh-Hurwitz criteria to study the synchronization behavior of identical planar magnetic binaries problem when the primaries are oblate spheroids. The designed controller of our own choice of the coefficient matrix of the error dynamics that satisfy the lyapunov stability and the RouthHurwitz criteria are found to be effective to achieve the synchronization between the magnetic binaries problem for our study. Numerical simulations are shown to verify the analytical results using mathematica. 


\section{The Equation of Motion}

The equation of motion of the planar magnetic-binaries problem by taking into consideration the oblateness of the primaries in a dimensionless, barycentric, pulsating rotating, co-ordinate system are as follows

$$
\begin{aligned}
\ddot{x}-f \dot{y} & =U_{x}, \\
\ddot{y}-f \dot{x} & =U_{y}, \\
\dot{x}^{2}+\dot{y}^{2} & =2 U-C \quad(C \text { is constant }),
\end{aligned}
$$

where

$$
\begin{aligned}
f= & 2 \omega-\left(\frac{1}{r_{1}^{3}}+\frac{I_{1}}{2 \mu_{1} r_{1}^{5}}+\frac{\lambda}{r_{2}^{3}}+\frac{\lambda I_{2}}{2 \mu_{2} r_{2}^{5}}\right), \\
U= & \frac{1}{2} \omega^{2}\left(x^{2}+y^{2}\right)+\frac{\omega}{\mu_{1}}\left\{( ( x ^ { 2 } + y ^ { 2 } ) - \mu _ { 2 } x ) \left(\frac{\mu_{1}}{r_{1}^{3}}\right.\right. \\
& \left.\left.+\frac{I_{1}}{2 r_{1}^{5}}\right)\right\}+\frac{\omega \lambda}{\mu_{2}}\left\{\left(\left(x^{2}+y^{2}\right)+\mu_{1} x\right)\left(\frac{\mu_{2}}{r_{2}^{3}}+\frac{I_{2}}{2 r_{2}^{5}}\right)\right\} \\
& +\frac{\mu_{1}}{r_{1}}+\frac{I_{1}}{2 r_{1}^{3}}+\frac{\mu_{1}}{r_{2}}+\frac{I_{2}}{2 r_{2}^{3}} .
\end{aligned}
$$

\section{Assumptions:}

1. Primaries participate in the circular motion around their center of mass.

2. Position vector of $\mathrm{P}$ at any time $\mathrm{t}$ be $\bar{r}=(x i+y j+z k)$ referred to a rotating frame of reference $\mathrm{O}(\mathrm{x}, \mathrm{y}, \mathrm{z})$ which is rotating with the same angular velocity $\omega$ as those the primaries.

3. Initially the primaries lie on the x-axis.

4. If the distance between the primaries as the unit of distance and the coordinate of one primary is $(\mu, 0,0)$ then the other is $(\mu-1,0,0)$.

5. The sum of their masses as the unit of mass. If mass of the one primaries $\mu$ then the mass of the other is $(1-\mu)$.

6. The unit of time in such a way that the gravitational constant $\mathrm{G}$ has the value unity and $q=m c$ where $c$ is the velocity of light. 


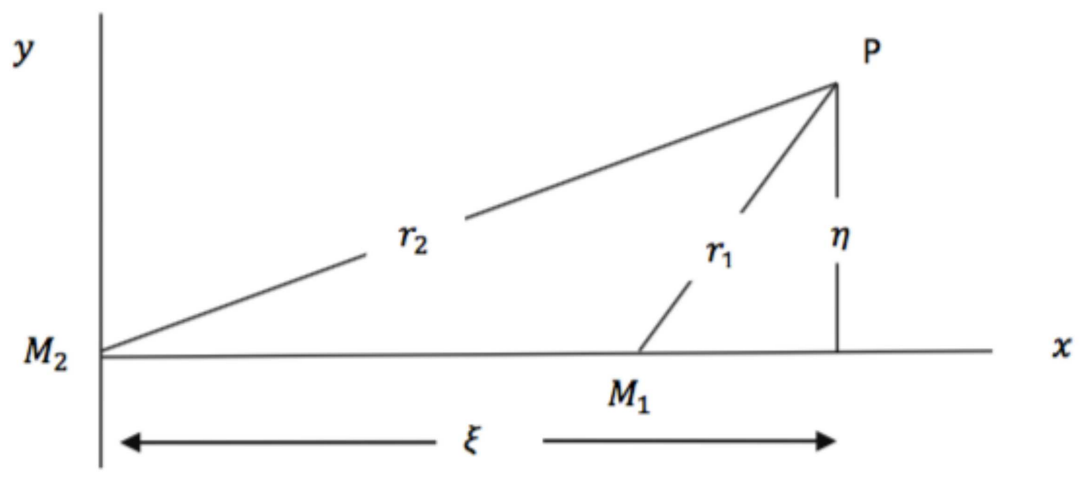

Figure 1

$$
r_{1}^{2}=(x-\mu)^{2}+y^{2}, \quad r_{2}^{2}=(x+1-\mu)^{2}+y^{2}, \quad \lambda=\frac{M_{2}}{M_{1}}
$$

( $M_{1}, M_{2}$ are the magnetic moment of the primaries which lies perpendicular to the plane of the motion.) By making some assumptions and transferring the origin of the coordinate system to the second mass Figure 1 the equations of motion (1) and (2), become

$$
\begin{aligned}
\ddot{\xi}-f \dot{\eta}= & U_{\xi}, \\
\ddot{\eta}+f \dot{\xi}= & U_{\eta}, \\
U_{\xi}= & \frac{\partial U}{\partial \xi}, \\
U_{\eta}= & \frac{\partial U}{\partial \eta}, \\
f= & 2 \omega-\left(\frac{1}{r_{1}^{3}}+\frac{I_{1}}{2 \mu_{1} r_{1}^{5}}+\frac{\lambda}{r_{2}^{3}}+\frac{\lambda I_{2}}{2 \mu_{2} r_{2}^{5}}\right), \\
U= & \frac{1}{2} \omega^{2}\left((\xi+\mu-1)^{2}+\eta^{2}\right) \\
& +\frac{\omega}{(1-\mu)}\left\{\left((\xi+\mu-1)^{2}+\eta^{2}-\mu(\xi+\mu-1)\right)\left(\frac{(1-\mu}{r_{1}^{3}}+\frac{I_{1}}{2 r_{1}^{5}}\right)\right\} \\
& +\frac{\omega \lambda}{\mu}\left\{\left((\xi+\mu-1)^{2}+\eta^{2}+(1-\mu)(\xi+\mu-1)\right)\left(\frac{\mu}{r_{2}^{3}}+\frac{I_{2}}{2 r_{2}^{5}}\right)\right\} \\
& +\frac{(1-\mu)}{r_{1}}+\frac{I_{1}}{2 r_{1}^{3}}+\frac{\mu}{r_{2}}+\frac{I_{2}}{2 r_{2}^{3}},
\end{aligned}
$$




$$
\begin{aligned}
& r_{1}^{2}=(\xi-1)^{2}+\eta^{2}, \\
& r_{2}^{2}=\xi^{2}+\eta^{2},
\end{aligned}
$$

and the new jacobi constant $C_{n}$ is given by

$$
\dot{\xi}^{2}+\dot{\eta}^{2}=2 U-C_{n}
$$

\section{Synchronization via Active Control}

Let

$$
\xi=x_{1}, \quad \dot{\xi}=x_{2}, \quad \eta=x_{3}, \quad \dot{\eta}=x_{4} .
$$

Then the equation (5) and (6) can be written as:

$$
\left\{\begin{aligned}
\dot{x_{1}}= & x_{2}, \\
\dot{x_{2}}= & x_{4} f_{2}+\left(x_{1}+\mu-1\right) \omega^{2}+\frac{\omega}{(1-\mu)}\left[\left\{2\left(x_{1}+\mu-1\right)-\mu\right\}\left\{\frac{(1-\mu)}{r_{1}^{3}}+\frac{I_{1}}{2 r_{1}^{5}}\right\}\right] \\
& -\left[\left\{\left(x_{1}+\mu-1\right)^{2}+x_{3}^{2}-\mu\left(x_{1}+\mu-1\right)\right\}\left\{\frac{3(1-\mu)\left(x_{1}-1\right)}{r_{1}^{5}}+\frac{5 I_{1}\left(x_{1}-1\right)}{2 r_{1}^{7}}\right\}\right] \\
& +\frac{\omega \lambda}{\mu}\left[\left\{2\left(x_{1}+\mu-1\right)+(1-\mu)\right\}\left\{\frac{(\mu}{r_{2}^{3}}+\frac{I_{2}}{2 r_{2}^{5}}\right\}\right] \\
& -\left[\left\{\left(x_{1}+\mu-1\right)^{2}+x_{3}^{2}-(1-\mu)\left(x_{1}+\mu-1\right)\right\}\left\{\frac{3 \mu x_{1}}{r_{2}^{5}}+\frac{5 I_{2} x_{1}}{2 r_{2}^{7}}\right\}\right] \\
& -\frac{(1-\mu)\left(x_{1}-1\right)}{r_{1}^{3}}-\frac{3 I_{2}\left(x_{1}-1\right)}{2 r_{1}^{5}}-\frac{\mu x_{1}}{r_{2}^{3}}+\frac{3 I_{2} x_{1}}{2 r_{2}^{5}}, \\
\dot{x_{3}}= & x_{4}, \quad-\frac{2 x_{3} \omega}{\dot{x}_{4}=-}\left\{\frac{(1-\mu)}{r_{1}^{3}}+\frac{I_{1}}{2 r_{1}^{5}}\right\} \\
& -\frac{x_{3} \omega}{(1-\mu)}\left[\left\{\left(x_{1}+\mu-1\right)^{2}+x_{3}^{2}-\mu\left(x_{1}+\mu-1\right)\right\}\left\{\frac{3(1-\mu)}{r_{1}^{5}}+\frac{5 I_{1}}{2 r_{1}^{7}}\right\}\right] \\
& +\frac{2 x_{3} \omega \lambda}{\mu}\left(\frac{\mu}{r_{2}^{3}}+\frac{I_{2}}{2 r_{2}^{5}}\right)-\frac{x_{3} \omega \lambda}{\mu}\left\{\left(x_{1}+\mu-1\right)^{2}+x_{3}^{2}+(1-\mu)\left(x_{1}+\mu-1\right)\right\} \\
& \left(\frac{3 \mu}{r_{2}^{5}}+\frac{5 I_{2}}{2 r_{2}^{7}}\right)-\frac{(1-\mu) x_{3}}{r_{1}^{3}}-\frac{3 I_{1} x_{3}}{2 r_{1}^{5}}-\frac{\mu x_{3}}{r_{2}^{3}}-\frac{3 I_{2} x_{3}}{2 r_{2}^{5}},
\end{aligned}\right.
$$

where

$$
r_{1}^{2}=\left(x_{1}-1\right)^{2}+x_{3}^{2}, \quad r_{2}^{2}=x_{1}^{2}+x_{3}^{2} .
$$


Corresponding to master system (9), the identical slave system is defined as:

$$
\left\{\begin{aligned}
\dot{y_{1}} & =y_{2}+u_{1}(t), \\
\dot{y}_{2} & =y_{4} f_{1}+\omega^{2}\left(y_{1}+\mu-1\right)+\frac{\omega}{(1-\mu)}\left[\left\{2\left(y_{1}+\mu-1\right)-\mu\right\}\left\{\frac{1-\mu}{r_{1}^{3}}+\frac{I_{1}}{2 r_{1}^{5}}\right\}\right. \\
& -\left\{\left(y_{1}+\mu-1\right)^{2}+y_{3}^{2}-\mu\left(y_{1}+\mu-1\right)\right\}\left\{\frac{3(1-\mu)\left(y_{1}-1\right)}{r_{1}^{5}}+\right. \\
& \left.\left.+\frac{5 I_{1}\left(y_{1}-1\right)}{2 r_{1}^{7}}\right\}\right]+\frac{\omega \lambda}{\mu}\left[\left\{2\left(y_{1}+\mu-1\right)+(1-\mu)\right\}\left\{\frac{\mu}{r_{2}^{3}}+\frac{I_{2}}{2 r_{2}^{5}}\right\}\right. \\
& \left.-\left\{\left(y_{1}+\mu-1\right)^{2}+y_{3}^{2}+(1-\mu)\left(y_{1}+\mu-1\right)\right\}\left\{\frac{3 \mu y_{1}}{r_{2}^{5}}+\frac{5 I_{2} y_{1}}{2 r_{2}^{7}}\right\}\right] \\
& -\frac{(1-\mu)\left(y_{1}-1\right)}{r_{1}^{3}}-\frac{3 I_{1}\left(y_{1}-1\right)}{r_{1}^{5}}-\frac{\mu y_{1}}{r_{2}}-\frac{3 I_{2} y_{1}}{r_{2}^{5}}+u_{2}(t), \\
\dot{y_{3}} & =y_{4}+u_{3}(t), \\
\dot{y_{4}} & =-y_{2} f_{3}+y_{3} \omega^{2}+\frac{2 y_{3} \omega}{(1-\mu)}\left(\frac{1-\mu}{r_{1}^{3}}+\frac{I_{1}}{2 r_{1}^{5}}\right) \\
& -\frac{y_{3} \omega}{(1-\mu)}\left[\left\{\left(y_{1}+\mu-1\right)^{2}+y_{3}^{2}-\mu\left(y_{1}+\mu-1\right)\right\}\left\{\frac{3(1-\mu)}{r_{1}^{5}}+\frac{5 I_{1}}{2 r_{1}^{7}}\right\}\right] \\
& +\frac{2 y_{3} \omega \lambda}{\mu}\left(\frac{\mu}{r_{2}^{3}}+\frac{I_{2}}{2 r_{2}^{5}}\right) \\
& -\frac{y_{3} \omega \lambda}{\mu}\left[\left\{\left(y_{1}+\mu-1\right)^{2}+y_{3}^{2}+(1-\mu)\left(y_{1}+\mu-1\right)\right\}\left\{\frac{3 \mu}{r_{2}^{5}}+\frac{5 I_{2}}{2 r_{2}^{7}}\right\}\right] \\
& -\frac{(1-\mu) y_{3}}{r_{1}^{3}}-\frac{3 I_{1} y_{3}}{2 r_{1}^{5}}-\frac{\mu y_{3}}{r_{2}^{3}}-\frac{3 I_{2} y_{3}}{2 r_{2}^{5}}+u_{4}(t),
\end{aligned}\right.
$$

where

$$
r_{1}^{2}=\left(y_{1}-1\right)^{2}+y_{3}^{2}, \quad r_{2}^{2}=y_{1}^{2}+y_{3}^{2},
$$

and $u_{i}(t) ; i=1,2,3,4$ are control functions to be determined. 
Let $e_{i}=y_{i}-x_{i} ; i=1,2,3,4$ be the synchronization errors. From (9) and (10), we obtain the error dynamics as follows:

$$
\begin{aligned}
& \dot{e_{1}}=\dot{y_{1}}-\dot{x_{1}}, \quad \dot{e_{2}}=\dot{y_{2}}-\dot{x_{2}}, \quad \dot{e_{3}}=\dot{y_{3}}-\dot{x_{3}}, \quad \dot{e_{4}}=\dot{y_{4}}-\dot{x_{4}}, \\
& \dot{e_{1}}=e_{2}+u_{1}(t) \text {, } \\
& \dot{e_{2}}=2 e_{4} \omega+y_{4} f_{1}-x_{4} f_{2}+e_{1} \omega^{2}+\frac{\omega}{(1-\mu)}\left[\left\{2\left(y_{1}+\omega-1\right)-\mu\right\}\left\{\frac{1-\mu}{r_{1}^{3}}+\frac{I_{1}}{2 r_{1}^{5}}\right\}\right. \\
& -\left\{\left(y_{1}+\mu-1\right)^{2}+y_{3}^{2}-\mu\left(y_{1}+\mu-1\right)\right\}\left\{\frac{3(1-\mu)\left(y_{1}-1\right)}{r_{1}^{5}}\right. \\
& \left.\left.+\frac{5 I_{1}\left(y_{1}-1\right)}{2 r_{1}^{7}}\right\}\right]+\frac{\omega \lambda}{\mu}\left[\left\{2\left(y_{1}+\mu-1\right)+(1-\mu)\right\}\left\{\frac{\mu}{r_{2}^{3}}+\frac{I_{2}}{2 r_{2}^{5}}\right\}\right. \\
& \left.-\left\{\left(y_{1}+\mu-1\right)^{2}+y_{3}^{2}+(1-\mu)\left(y_{1}+\mu-1\right)\right\}\left\{\frac{\left(3 \mu y_{1}\right)}{r_{2}^{5}}+\frac{\left.5 I_{2} y_{1}\right)}{2 r_{2}^{7}}\right\}\right] \\
& -\frac{(1-\mu)\left(y_{1}-1\right)}{r_{1}^{3}}-\frac{3 I_{1}\left(y_{1}-1\right)}{r_{1}^{5}}-\frac{\mu y_{1}}{r_{2}}-\frac{3 I_{2} y_{1}}{r_{2}^{5}} \\
& -\left[\frac { \omega } { ( 1 - \mu ) } \left[\left\{2\left(x_{1}+\mu-1\right)-\mu^{2}\right\}\left\{\frac{1-\mu}{r_{1}^{3}}+\frac{I_{1}}{2 r_{1}^{5}}\right\}\right.\right. \\
& -\left\{\left(x_{1}+\mu-1\right)^{2}+x_{3}^{2}-\mu\left(x_{1}+\mu-1\right)\right\}\left\{\frac{3(1-\mu)\left(x_{1}-1\right)}{r_{1}^{5}}\right. \\
& \left.\left.+\frac{5 I_{1}\left(x_{1}-1\right)}{2 r_{1}^{7}}\right\}\right]+\frac{\omega \lambda}{\mu}\left[\left\{2\left(x_{1}+\mu-1\right)+(1-\mu)\right\}\left\{\frac{\mu}{r_{2}^{3}}+\frac{I_{2}}{2 r_{2}^{5}}\right\}\right. \\
& \left.-\left\{\left(x_{1}+\mu-1\right)^{2}+x_{3}^{2}+(1-\mu)\left(x_{1}+\mu-1\right)\right\}\left\{\frac{\left(3 \mu x_{1}\right)}{r_{2}^{5}}+\frac{\left.5 I_{2} x_{1}\right)}{2 r_{2}^{7}}\right\}\right] \\
& \left.-\frac{(1-\mu)\left(x_{1}-1\right)}{r_{1}^{3}}-\frac{3 I_{1}\left(x_{1}-1\right)}{r_{1}^{5}}-\frac{\mu x_{1}}{r_{2}}-\frac{3 I_{2} x_{1}}{r_{2}^{5}}\right]+u_{2}(t), \\
& \dot{e_{3}}=e_{4}+u_{3}(t) \text {, } \\
& \dot{e_{4}}=-2 e_{2} \omega+y_{2} f_{3}+x_{2} f_{4}+e_{3} \omega^{2}+\frac{2 y_{3} \omega}{(1-\mu)}\left\{\frac{1-\mu}{r_{1}^{3}}+\frac{I_{1}}{2 r_{1}^{5}}\right\} \\
& -\frac{y_{3} \omega}{(1-\mu)}\left[\left\{\left(y_{1}+\mu-1\right)^{2}+y_{3}^{2}-\mu\left(y_{1}+\mu-1\right)\right\}\left\{\frac{3(1-\mu)}{r_{1}^{5}}+\frac{5 I_{1}}{2 r_{1}^{7}}\right\}\right] \\
& +\frac{2 y_{3} \omega \lambda}{\mu}\left(\frac{\mu}{r_{2}^{3}}+\frac{I_{2}}{2 r_{2}^{5}}\right) \\
& -\frac{y_{3} \omega \lambda}{\mu}\left[\left\{\left(y_{1}+\mu-1\right)^{2}+y_{3}^{2}+(1-\mu)\left(y_{1}+\mu-1\right)\right\}\left\{\frac{3 \mu}{r_{2}^{5}}+\frac{5 I_{2}}{2 r_{2}^{7}}\right\}\right] \\
& -\frac{(1-\mu) y_{3}}{r_{1}^{3}}-\frac{3 I_{1} y_{3}}{2 r_{1}^{5}}-\frac{\mu y_{3}}{r_{2}^{3}}-\frac{3 I_{2} y_{3}}{2 r_{2}^{5}} \\
& -\left[\frac{2 x_{3} \omega}{(1-\mu)}\left\{\frac{1-\mu}{r_{1}^{3}}+\frac{I_{1}}{2 r_{1}^{5}}\right\}\right. \\
& -\frac{x_{3} \omega}{(1-\mu)}\left[\left\{\left(x_{1}+\mu-1\right)^{2}+x_{3}^{2}-\mu\left(x_{1}+\mu-1\right)\right\}\left\{\frac{3(1-\mu)}{r_{1}^{5}}+\frac{5 I_{1}}{2 r_{1}^{7}}\right\}\right] \\
& +\frac{2 x_{3} \omega \lambda}{\mu}\left\{\frac{\mu}{r_{2}^{3}}+\frac{I_{2}}{2 r_{2}^{5}}\right\} \\
& -\frac{x_{3} \omega \lambda}{\mu}\left[\left\{\left(x_{1}+\mu-1\right)^{2}+x_{3}^{2}+(1-\mu)\left(x_{1}+\mu-1\right)\right\}\left\{\frac{3 \mu}{r_{2}^{5}}+\frac{5 I_{2}}{2 r_{2}^{7}}\right\}\right] \\
& \left.-\frac{(1-\mu) x_{3}}{r_{1}^{3}}-\frac{3 I_{1} x_{3}}{2 r_{1}^{5}}-\frac{\mu x_{3}}{r_{2}^{3}}-\frac{3 I_{2} x_{3}}{2 r_{2}^{5}}\right]+u_{4}(t) \text {. }
\end{aligned}
$$

This above error system to be controlled is a linear system with control functions. Thus, let us redefine the control functions so that the terms in (11) 
which cannot be expressed as linear terms in $e_{i}^{\prime} s$ are eliminated:

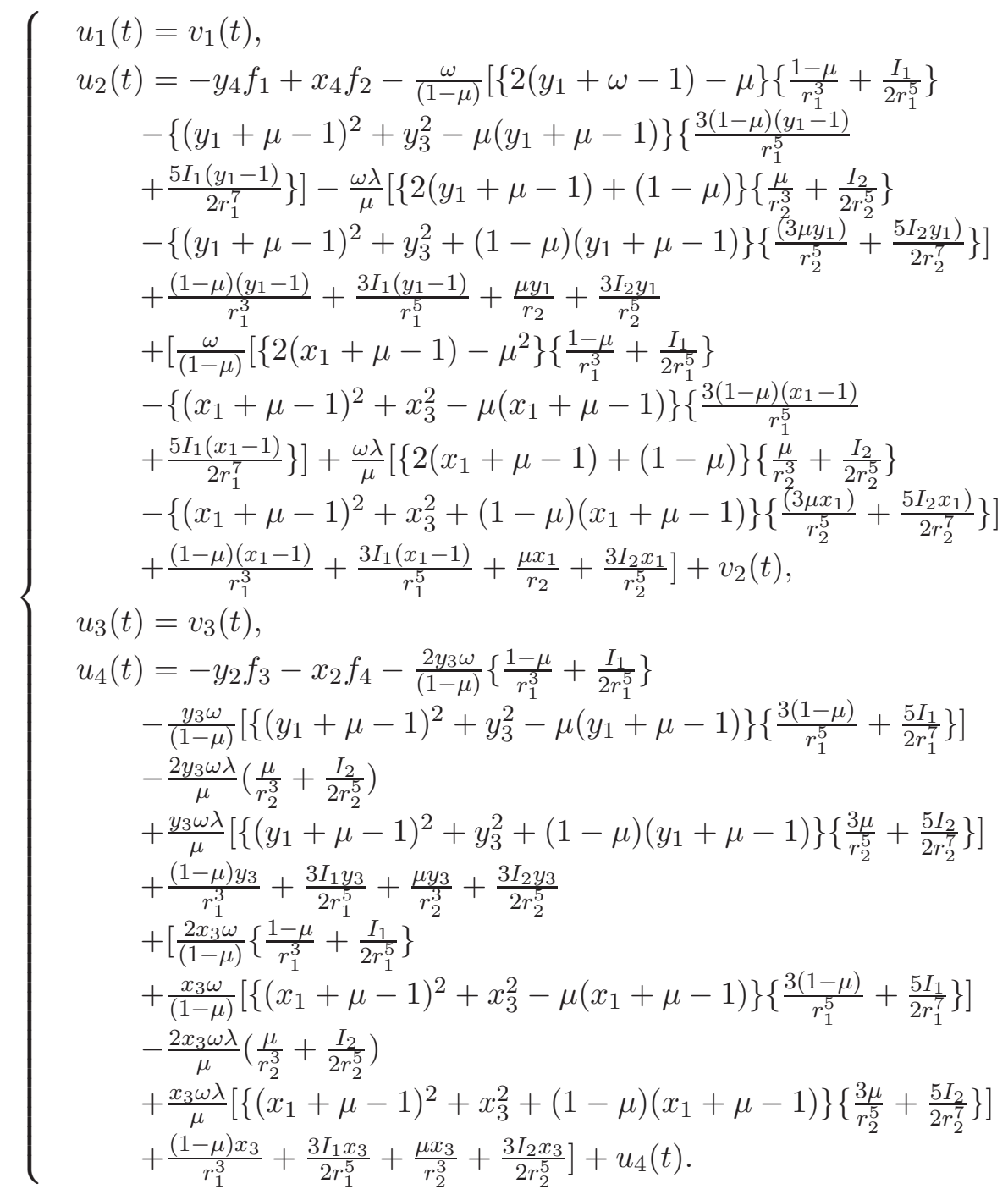


The new error system can be expressed as:

$$
\left\{\begin{array}{l}
\dot{e_{1}}=e_{2}+v_{1}(t), \\
\dot{e_{2}}=2 \omega e_{4}+e_{1} \omega^{2}+v_{2}(t), \\
\dot{e_{3}}=e_{4}+v_{3}(t), \\
\dot{e_{4}}=-2 \omega e_{2}+\omega e_{3}+v_{4}(t) .
\end{array}\right.
$$

The error system (13) to be controlled is a linear system with a control input $v_{i}(t)(i=1,4)$ as function of the error states $e_{i}(i=1,4)$. As long as these feedbacks stabilize the system $e_{i}(i=1,4)$ converge to zero as time t tends to infinity. This implies that master and the slave system are synchronized with active control. There are many possible choice for the control $v_{i}(t)(i=1,4)$. We choose

$$
\left[\begin{array}{l}
v_{1}(t) \\
v_{2}(t) \\
v_{3}(t) \\
v_{4}(t)
\end{array}\right]=A\left[\begin{array}{l}
e_{1} \\
e_{2} \\
e_{3} \\
e_{4}
\end{array}\right]
$$

Here $\mathrm{A}$ is a $4 \times 4$ constant matrix to be determined. As per Lyapunov stability theory and Routh-Hurwitz criterion, in order to make the closed loop system (14) stable, proper choice of elements of A has to be made so that the system (14) must have all eigen values with negative real parts. We choose

$$
A=\left[\begin{array}{cccc}
-1 & -1 & 0 & 0 \\
-\omega^{2} & -1 & 0 & -2 \omega \\
0 & 0 & -1 & -1 \\
0 & 2 \omega & -\omega & -1
\end{array}\right]
$$

and, define a matrix $\mathrm{B}$ as

$$
\left[\begin{array}{c}
\dot{e_{1}} \\
\dot{e_{2}} \\
\dot{e_{3}} \\
\dot{e_{4}}
\end{array}\right]=B\left[\begin{array}{l}
e_{1} \\
e_{2} \\
e_{3} \\
e_{4}
\end{array}\right],
$$

where

$$
B=\left[\begin{array}{cccc}
-1 & 0 & 0 & 0 \\
0 & -1 & 0 & 0 \\
0 & 0 & -1 & 0 \\
0 & 0 & 0 & -1
\end{array}\right]
$$




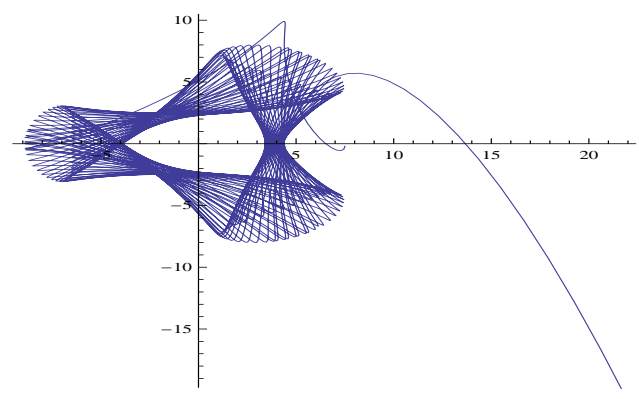

Figure 2

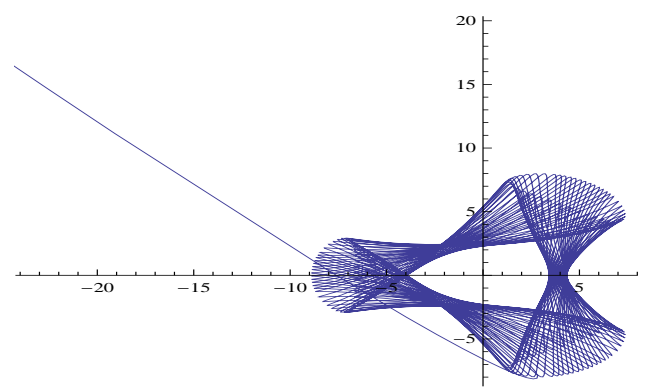

Figure 3

Clearly, B has eigen values with negative real parts. This implies $\lim _{t \rightarrow \infty}\left|e_{i}\right|=$ $0 ; i=1,2,3,4$ and hence, complete synchronization is achieved between the master and slave systems (9) and (10) respectively.

\section{Numerical Simulation}

We select the parameters $\mu=0.0968101$ and $\lambda=5$ with the initial conditions for master and slave systems $\left[x_{1}(0)=6.5, x_{2}(0)=0.8, x_{3}(0)=-6.5, x_{4}(0)=0.2\right]$ and $\left[y_{1}(0)=7.5, y_{2}(0)=2.1, y_{3}(0)=-.2, y_{4}(0)=1.2\right]$ respectively. We have simulated the system under consideration using Mathematica Phase portraits and analysis of master and slave system are the witness of irregular behavior of the system (Figures 2 and 3 ).

Simulation results for uncoupled system are presented in Figures 4,5 and 6 and that of controlled system are shown in Figures 7,8 and 9 respectively. 


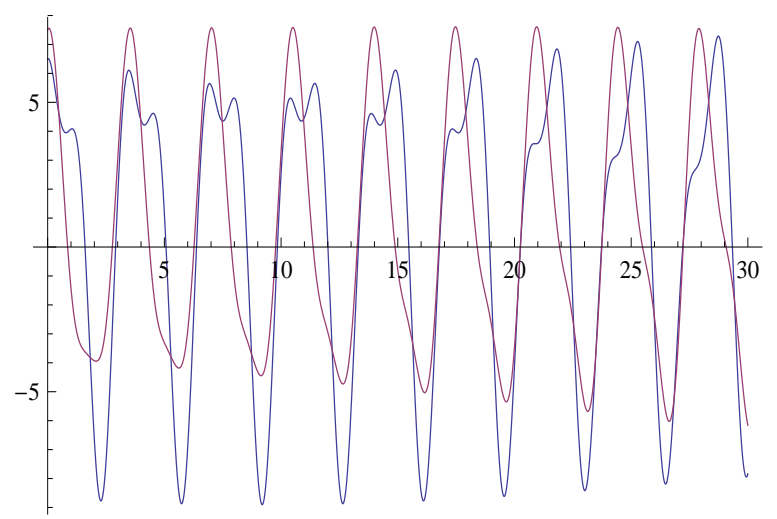

Figure 4

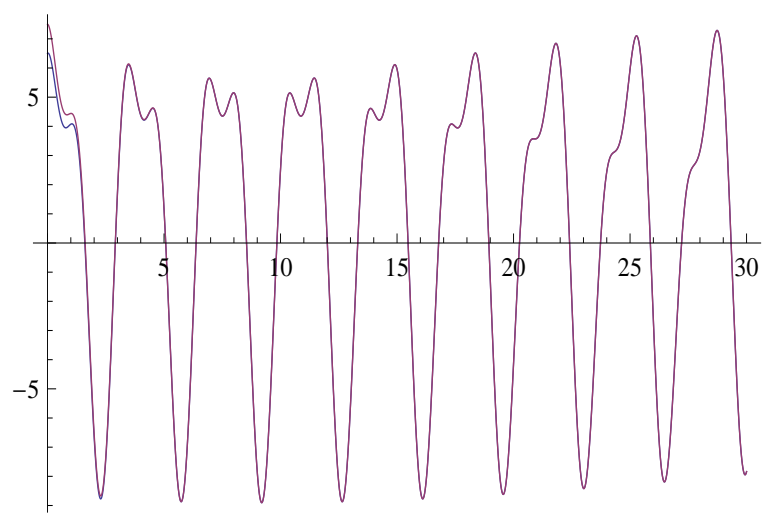

Figure 5

\section{Conclusion}

An investigation on synchronization in the planar magnetic-binaries problem by taking into consideration the oblateness of primaries via active control technique based on Lyapunov stability theory and Routh-Hurwitz criteria have been made. The results were validated by numerical simulations using Mathematica.

\section{References}

[1] Ott E., Grebogi C., Yorke J. A., Controlling Chaos, Physics Rev. Lett., 64 (1990), 11961199.

[2] Pecora L. M., Carroll T. L., Synchronization in chaotic systems, Physics Rev. Lett., 64 (1990), 821-824. 


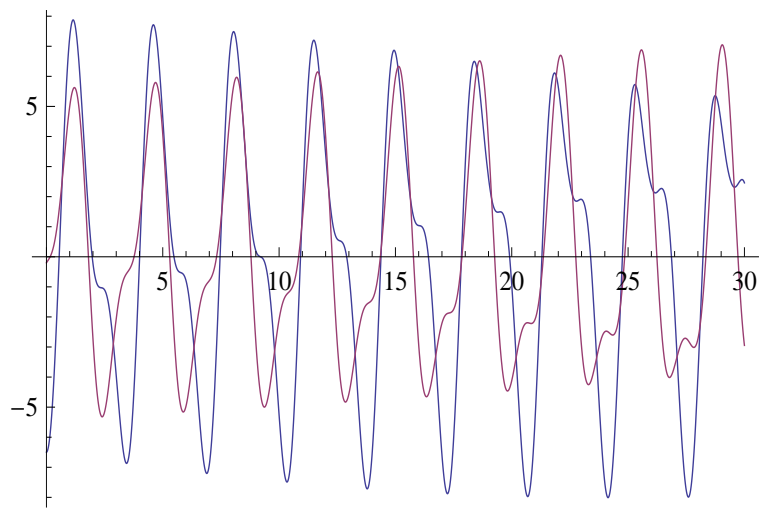

Figure 6

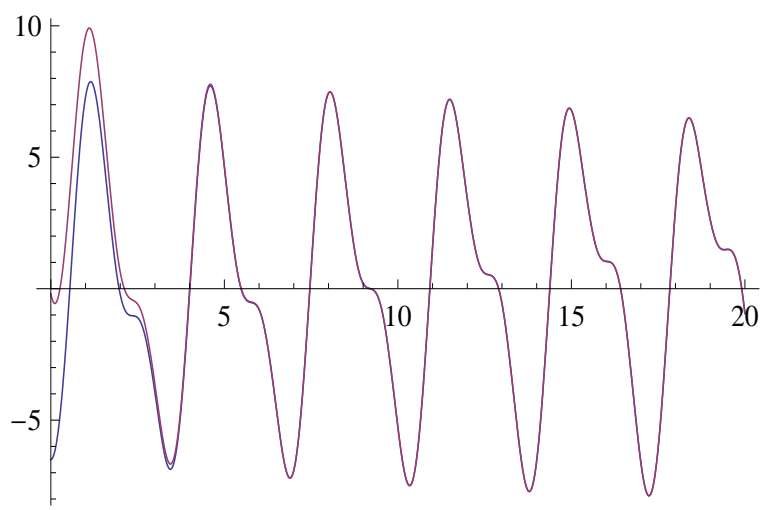

Figure 7

[3] Lu L., Zhang C., Guo Z. A., Synchronization between two diffeerent chaotic systems with nonlinear feedback control, Chinese Physics, 16-6 (2007), 1603-1067.

[4] Haeri M., Emadzadeh A., Synchronizing different chaotic systems using active sliding mode control, Chaos,Solitonsand Fractals, 1 (2007), 119-129.

[5] Wang Y., Guan Z. H., Wang H. O., Feedback and adaptive control for the synchronization of Chen system via a single variable, Physics Letters 312-1 (2003), 30-40.

[6] Park J. H., Chaos synchronization between two different chaotic dynamical systems, Chaos, Solitons and Fractals, 27-2 (2006), 549-554.

[7] Bai E.W., Lonngren K.E., Synchronization of two Lorenz systems using active control, Chaos, Solitons and Fractals, 8 (1997), 51-58.

[8] Wang C., He Y., Ma J., Long H., Parameters Estimation, Mixed Synchronization, and Antisynchronization in Chaotic Systems, Complexity, 20-1 (2014).

[9] Stormer C.F., Arch. Sci. Phys. et. Nat. Geneva, 1907, 24-150. 


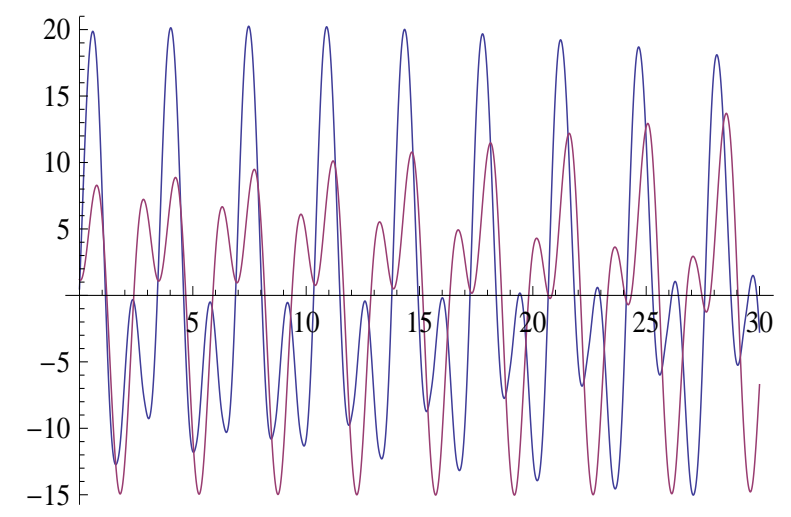

Figure 8

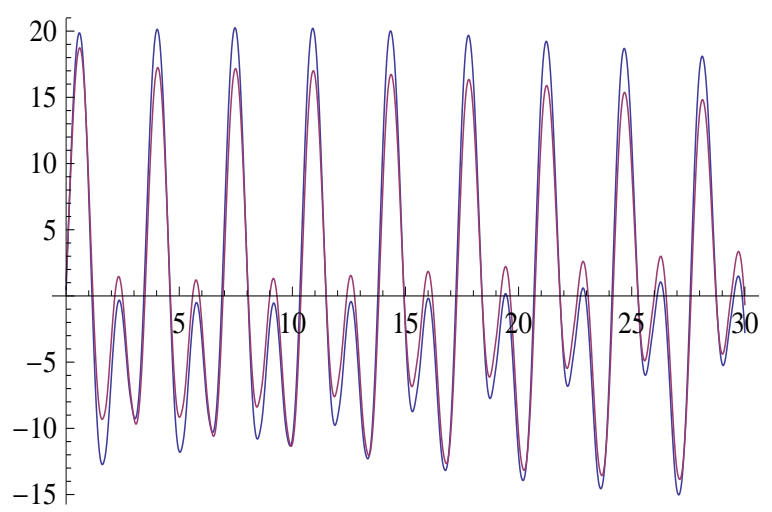

Figure 9

[10] Marvaganis A., The magnetic-binaries problem : motion of a charged particle in the region of a magnetic-binary system, Astrophys. space sci., 54 (1978), 305-313.

[11] Marvaganis A., Stationary solutions and their stability in the magnetic-binary problem when the primaries are oblate spheroids, Astron. Astrophys., 80 (1979), 130-133.

[12] Arif Mohd., Motion of a charged particle when the primaries are oblate spheroids, International Journal of Applied Math and Mech., 6 (4),2010,94-106.

[13] Khan A., Shahzad M., Synchronization of Circular Restricted Three Body Problem with Lorenz Hyper Chaotic System Using a Robust Adaptive Sliding Mode Controller, Complexity 18-6 (2013). 
